\title{
Interfacial Adsorption State of Protonated Lipophilic Porphyrins in a Liquid-Liquid System by Using Reflection Spectrometry
}

\author{
Yoshio MoriYa, ${ }^{\dagger}$ Shinichi NaKata, Hirotaka Morimoto, and Nobuaki Ogawa \\ Faculty of Engineering and Resource Science, Akita University, 1-1 Tegata Gakuen-cho, Akita 010-8502, Japan
}

\begin{abstract}
Analysis by reflection spectrometry was performed to clarify the interfacial adsorption of protonated lipophilic tetraphenylporphyrin derivatives in a dodecane-aqueous sulfuric acid system, and to confirm the utility of partial reflection spectroscopy. Interfacial adsorption was not observed for porphyrins substituted at the 2,6 positions of mesophenyl groups, suggesting that the substituents prevent porphyrins from forming aggregates by steric hindrance. Polymorphous J-aggregates of acid dications were produced by tetra-p-tolylporphyrin with a saturated interfacial molecular density of $1.0 \times 10^{-10} \mathrm{~mol} \mathrm{~cm}{ }^{-2}$, which could yield $48^{\circ}$ as a mean tilting angle of the pyrrole ring plane from the interface normal. Partial-reflection spectrometry can provide sensitive detection and molecular orientation analysis of interfacial adsorbates.
\end{abstract}

(Received July 30, 2004; Accepted September 10, 2004)

\section{Introduction}

The liquid-liquid interface has received a great deal of attention in connection with phase-transfer catalysis, ${ }^{1}$ separation chemistry $^{2}$ and biochemical sciences. ${ }^{3}$ This is because the liquid-liquid interface provides a unique chemical reaction field in which molecular species are spontaneously trapped to generate organized molecules with an orientation that is not available in a bulk solution. To understand the interfacial adsorption states, several spectroscopic techniques have been developed, such as two-phase transmission spectrometry, represented by the centrifugal liquid membrane (CLM) method, ${ }^{4}$ attenuated total-internal reflection (ATR) spectrometry, ${ }^{5,6}$ total internal-reflection fluorometry, ${ }^{7}$ resonance Raman spectrometry, ${ }^{8,9}$ and second-harmonic generation (SHG) spectrometry. ${ }^{10}$

We have contributed to develop reflection spectrometry in connection with a direct measurement of the acid dication of meso-tetraphenylporphyrin (TPP) in an aggragate $\left(\mathrm{H}_{2} \mathrm{TPPX}_{2}\right)_{n, \text {, }}$ formed at the inert organic solvent/aqueous inorganic acid (HX) interface. ${ }^{11-13}$ It was found in the toluene-aqueous sulfuric acid system that external reflection (ER) spectrometry, where incident light was irradiated obliquely on the interface from the aqueous phase to the organic phase, could provide not only a single spectrum of interfacial adsorbate with no interference of organic bulk phase, but also qualitative knowledge about the molecular orientation by observing positive/negative bands in the $p$-polarized ER ( $p$-ER) spectrum. ${ }^{12}$ In the dodecane-aqueous sulfuric acid system, all of the free base TPP was protonated to produce a J-aggregate, and the interfacial adsorption state changed from a monolayer form to a multilayer one similar to precipitation with an increasing amount of TPP. Further, it was found that $p$-polarized partial internal reflection ( $p$-PIR) spectrometry could also yield positive/negative bands with the

† To whom correspondence should be addressed.

E-mail: ymoriya@ipc.akita-u.ac.jp same band locations, but with reversed sign, as found in the $p$ ER spectrum. ${ }^{13}$

These results and knowledge were obtained by using only one testing reagent TPP. While TPP is highly lipophilic due to having meso-phenyl group, the amount of its interfacial adsorbate tends to change by dissolution of the monomeric species in the aqueous phase. Therefore, it could be interesting to investigate the interfacial adsorption or precipitation by using other lipophilic porphyrins. In the present work, such interfacial phenomena were investigated in the cases of some available TPP derivatives in the dodecane-aqueous sulfuric acid system in order to confirm the interfacial adsorption of porphyrins, and to clarify the utility of partial reflection spectrometry, including ER and PIR techniques.

\section{Experimental}

Reagents and chemicals

TPP (Dojindo Laboratories) and its lipophilic derivatives (Tokyo Kasei Kogyo Co. Ltd.), including tetra- $p$-tolyl-, tetrakis(2,4,6-trimethylphenyl)-, tetrakis-(2,6-dichlorophenyl)-, and tetrakis-(pentafluorophenyl)-porphines, were used as received; the free base of porphyrin (P) for those are shown in Fig. 1 along with their abbreviations. Stock solutions of those were prepared by dissolving in dodecane (Wako Pure Chem. Co. Ltd.).

\section{Apparatus and procedure}

Two kinds of optical equipments were used for the present study; that is, a prism-cell device and a variable-angle device. The optical arrangements for the two devices are simply shown as (a) and (b), respectively, in Fig. 2. The prism-cell device, comprising a quartz liquid-cell (light path length: $2 \mathrm{~cm}$, cell width: $1 \mathrm{~cm}$ ) and a pair of quartz prisms $\left(30^{\circ}-30^{\circ}-120^{\circ}\right)$, was designed for the analysis of saturated interfacial adsorbates. By changing the height of the device, the measurement mode can be switched for the transmission absorption spectra of the 

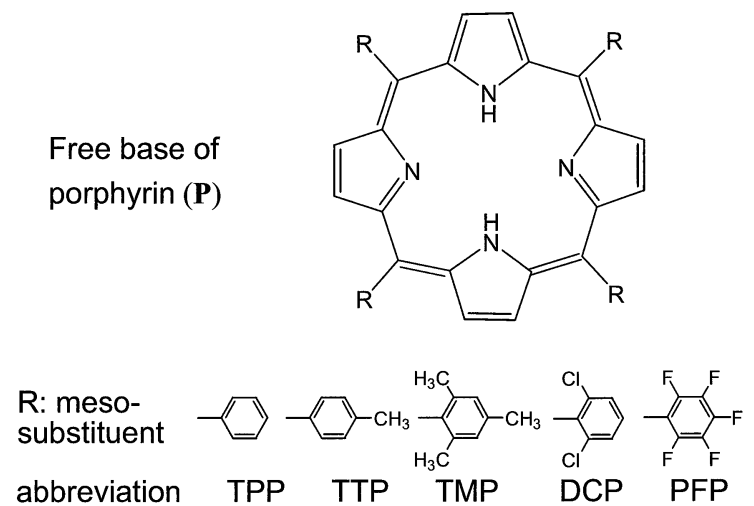

Fig. 1 Structures and abbreviations of the free-base porphyrins used in this study. (a)

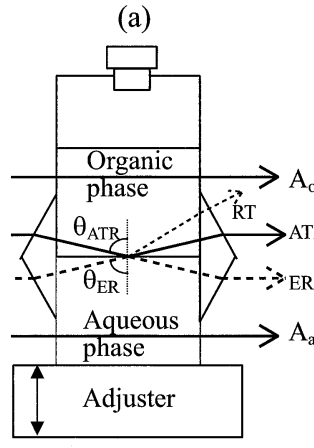

(b)

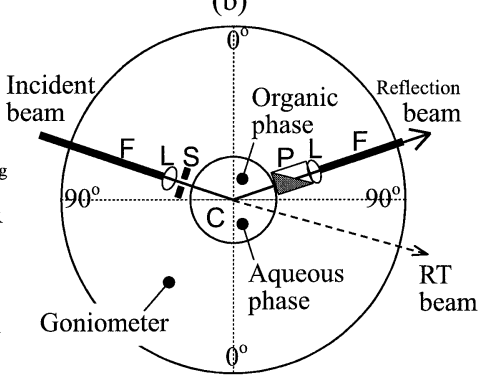

C: cylindrical cell, S: screen with an orifice F: optical fiber, L: terminal lens, P: polarizer
Fig. 2 Schematic illustrations of (a) a prism-cell device and (b) a variable-angle device. RT is the refracted transmission.

organic and aqueous phases $\left(A_{\text {org }}, A_{\text {aq }}\right)$ and the reflection spectra at the liquid-liquid interface (ER, ATR), which are dragged down with the corresponding blank spectra. The angle of incidence for the reflection spectrometry was estimated from the refractive indices of the quartz material (1.457) and the organic and aqueous media, which were experimentally determined by an Abbe refractometer (Erma Optical Works, Ltd.). Spectroscopic measurements were carried out on a double-beam UV-visible spectrometer (Perkin-Elmer, Model $\lambda 40$ ). Absorption spectra of dodecane solutions $\left(A_{\text {soln }}\right)$ for the free base of porphyrins were measured using an optical cell of $1 \mathrm{~cm}$ light path length at the beginning. In order to accelerate the interfacial adsorption reaction, a mixing-standing procedure was available. That is, a mixture of the same volume $\left(3 \mathrm{~cm}^{3}\right)$ of aqueous and organic solutions in a Teflon test tube was shaken (250 strokes/min) for $30 \mathrm{~min}$, centrifuged, transferred entirely into a prism-cell and allowed to left standing for 20 min until performing spectral measurements.

On the other hand, a variable-angle device was designed to allow measurements with transmission, ER, ATR and PIR geometries. Optical fibers with two focus lenses and a polarizer were assembled on a goniometer, and a cylindrical cell (capacity $8 \mathrm{~cm}^{3}$ with $2-\mathrm{cm}$ i.d. and $2.55 \mathrm{~cm}$ in length) was placed at the center. Spectroscopic measurements in this case were carried out by using a CCD array spectrometer (Ocean Optics, Model USB2000).

\begin{tabular}{|cll|}
\hline$(m+n) \mathbf{P}_{\mathrm{o}}$ & organic phase \\
\hline$+\rightleftharpoons(m+n) \mathrm{H}_{2} \mathbf{P X}_{2, \mathrm{i}} \rightleftharpoons\left(\mathrm{H}_{2} \mathbf{P} \mathbf{X}_{2}\right)_{\mathrm{n}, \mathrm{i}}$ & interface \\
\hline $2(m+n) \mathrm{HX}_{\mathrm{aq}}$ & $m \mathrm{H}_{2} \mathbf{P}^{2+}{ }_{\text {aq }}+2 m \mathrm{X}_{\text {aq }}$ & aqueous phase \\
\hline
\end{tabular}

Fig. 3 Interfacial protonation-association mechanism proposed for TPP in the dodecane-aqueous inorganic acid (HX) system. The free base of porphyrins is represented by $\mathbf{P}$.

\section{Results and Discussion}

Interfacial adsorption behavior of TPP derivatives

As for the TPP with a total concentration [TPP $]_{t}$ of $0.24 \mu \mathrm{M}$ $\left(1 \mathrm{M}=1 \mathrm{~mol} \mathrm{dm}^{-3}\right)$ in the dodecane- $4 \mathrm{M} \mathrm{H}_{2} \mathrm{SO}_{4}$ system, a Soret band at $416 \mathrm{~nm}$ of free base porphyrin disappeared in the organic phase spectrum to yield a diprotonated interfacial species, $\mathrm{H}_{2}$ TPPX $2, \mathrm{i}\left(\mathrm{X}: \mathrm{HSO}_{4}^{-}\right)$, which consequently changed to a J-aggregate, $\left(\mathrm{H}_{2} \mathrm{TPPX}_{2}\right)_{n, \mathrm{i}}$ at the interface and a monomeric ion, $\mathrm{H}_{2} \mathrm{TPP}^{2+}{ }_{\text {aq }}$, in the aqueous phase. Thus, we could observe the ATR and ER spectra of an aggregate having main three bands at 720, 473 and $412 \mathrm{~nm}$ within the visible wavelength region, as described previously. ${ }^{13}$ The interfacial protonation-association mechanism of TPP can be expressed as in Fig. 3, where free base of porphyrin is expressed by $\mathbf{P}$. It may be predictable that the same mechanism is found for the other lipophilic TPP derivatives as well. In practice, however, we could not find any reflection bands corresponding to interfacial adsorbates, nor an absorption band in the aqueous phase in the cases of TMP, DCP and PFP with $[\mathbf{P}]_{\mathrm{t}}=0.24 \mu \mathrm{M}$. The feature common to these three porphyrines is to have substituents at the 2,6 positions of the meso-phenyl group.

It is known that N-protonation induces a structural change; because that the free-base porphyrin converts from a configuration in which the aryl moiety twists relative to the macrocycle plane to one in which it is nearly coplanar, thus promoting aggregation. ${ }^{14}$ The 2,6-position substituents could prevent a phenyl group from revolving around the $\mathrm{C}$-phenyl axis, and also could prevent the $\mathrm{H}_{2} \mathbf{P} X_{2, \mathrm{i}}$ from associating by a steric hindrance. This explanation is supported by the fact that the aggregate formation was not observed even in the case of tetrakis-(3,5-dimethoxy-phenyl)-porphine. ${ }^{15} \quad$ Further, the enhanced hydrophobicity by halogen or methyl substitution may shift the equilibrations toward the left-hand side in Fig. 3. In fact, the initial concentration in the organic phase, $[\mathbf{P}]_{\mathrm{o}}$, was not decreased by contact with the acidic aqueous phase in the cases of TMP, DCP, and PFP.

Consequently, it was only the case of TTP that the reflection spectrum of protonated chemical species was clearly observed within the tested TPP derivatives. The species was observed in J-aggregate form, as in the case of TPP. Both TPP and TTP have no 2,6-position substituents in the meso-phenyl group. Inversely, this fact supports the above-mentioned consideration about the steric hindrance. Similar J-aggregate formations are also found for the other TPP derivatives having a substituent at the $p$-position of the meso-phenyl group, such as tetra-pnitrophenylporphyrin, tetra- $p$-methoxyphenylporphyrin, and so on. ${ }^{15}$

\section{Adsorption state of diprotonated TTP}

The reflection spectrum of diprotonated TTP in aggregate 


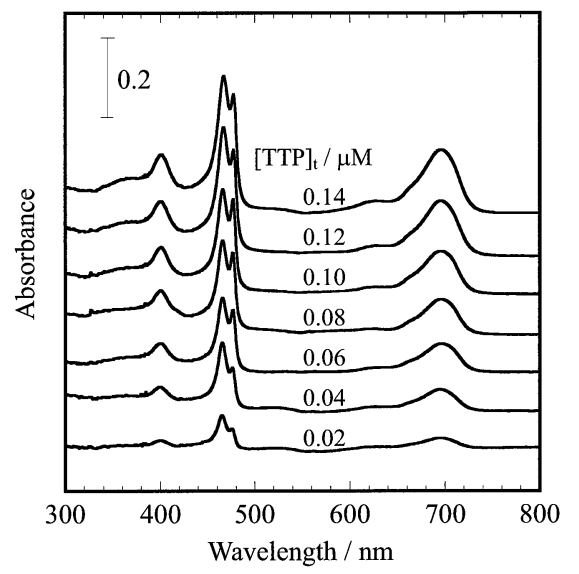

Fig. 4 ATR spectroscopic change of $\left(\mathrm{H}_{2} \mathrm{TPPX}_{2}\right)_{n, \mathrm{i}}$ accompanying an increase in $[\mathrm{TTP}]_{\mathrm{t}}$ at constant $\left[\mathrm{H}_{2} \mathrm{SO}_{4}\right]_{\mathrm{t}}=5 \mathrm{M}$.

form as $\left(\mathrm{H}_{2} \mathrm{TPPX}_{2}\right)_{n, \mathrm{i}}$ has several bands within the visible wavelength region, as mentioned bellow. The reflection absorbance for each of the bands increased as the concentration of sulfuric acid increased from 2 to $4 \mathrm{M}$. The Soret band at 418 $\mathrm{nm}$ for the free-base of $[\mathrm{TTP}]_{\mathrm{t}}=0.24 \mu \mathrm{M}$ in the organic phase disappeared entirely to yield a diprotonated species under the aqueous-phase condition of $\left[\mathrm{H}_{2} \mathrm{SO}_{4}\right]_{\mathrm{t}}=5 \mathrm{M}$ (refractive index: 1.38). Unlike the case of TPP, a monomeric species was not observed in the aqueous phase. Therefore, the ATR spectra at the angle of incidence, $\theta_{\mathrm{ATR}}=79.8^{\circ}$ (which is greater than the critical angle $\theta_{\mathrm{C}}=76.0^{\circ}$ ), as well as the ER spectra correspond only to interfacial adsorbates. Figure 4 shows the spectral changes of ATR with the variation of $[\mathrm{TTP}]_{\mathrm{t}}$ from 0.02 to 0.14 $\mu \mathrm{M}$ at constant $\left[\mathrm{H}_{2} \mathrm{SO}_{4}\right]_{\mathrm{t}}=5 \mathrm{M}$. The ATR absorbance of each band increased with the increase of $[\mathrm{TTP}]_{\mathrm{t}}$. The spectrum has four main bands at 695, 477, 466, and $400 \mathrm{~nm}$ together with some shoulder bands. ${ }^{16}$ Split bands at 477 and $466 \mathrm{~nm}$, and a broad band at $400 \mathrm{~nm}$ with a shoulder at around $360 \mathrm{~nm}$ correspond to the $473 \mathrm{~nm}$ and $412 \mathrm{~nm}$ bands of $\left(\mathrm{H}_{2} \mathrm{TPPX}_{2}\right)_{n, \mathrm{i}}$ in the J-aggregate, respectively. Sharp and intense absorption bands at $473 \mathrm{~nm}$ could arise from the dipole moment polarized along the long axis with the aid of an interporphyrin charge resonance in the excited states, whereas a broad absorption band at $412 \mathrm{~nm}$ could arise from the moment along the short axis. ${ }^{13,17}$ Therefore, split bands at 477 and $466 \mathrm{~nm}$ observed in the present system indicate the coexistence of two kinds of $\mathrm{J}$ aggregates $\left(\mathrm{J}_{1}\right.$ and $\left.\mathrm{J}_{2}\right)$ with a polymorphous relation.

The ATR absorbance for each of the four main bands was plotted against the interfacial molecular density, $\left[\mathrm{H}_{2} \mathrm{TTPX}\right]_{\mathrm{i}}$, as shown in Fig. 5. The absorbance at $477 \mathrm{~nm}$, which may belong to the $\mathrm{J}_{1}$-aggregate, increased in proportion to $\left[\mathrm{H}_{2} \mathrm{TTPX}_{2}\right]_{\mathrm{i}}$, and slowly increased along another line with a different slope after passing an inflection point. This must arise from a change of the reflection coefficient along with the change of the interfacial adsorption state from the monolayer form to the multilayer one, as indicated by diprotonated TPP in the dodecane- $4 \mathrm{M} \mathrm{H}_{2} \mathrm{SO}_{4}$ system. ${ }^{13}$ However, the increasing tendency of the $466 \mathrm{~nm}$ band, which may belong to the $\mathbf{J}_{2}$-aggregate, changed along a loose curve near the inflection point. This suggests that the $\mathrm{J}_{2^{-}}$ aggregate formation is depressed by the smooth $\mathrm{J}_{1}$-aggregate formation. The common inflection point at $\left[\mathrm{H}_{2} \mathrm{TTPX}_{2}\right]_{\mathrm{i}}=1.0 \times$ $10^{-10} \mathrm{~mol} \mathrm{~cm}^{-2}$ in Fig. 5 must correspond to the saturated interfacial molecular density in a monolayer comprising $\mathrm{J}_{1}$ and $\mathbf{J}_{2}$. The interfacial adsorption state could transform from monolayer to multilayer (interfacial precipitation) when beyond

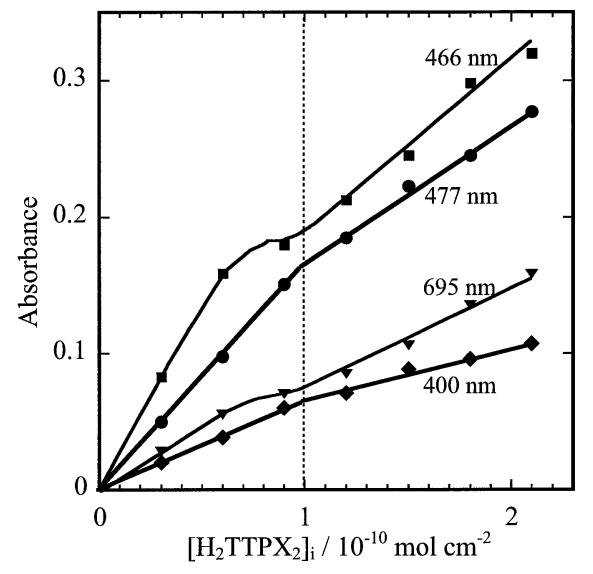

Fig. 5 Plots of the ATR absorbance vs. interfacial molecular density represented by $\left[\mathrm{H}_{2} \mathrm{TPPX}_{2}\right]_{\mathrm{i}} / \mathrm{mol} \mathrm{cm}^{-2}$.

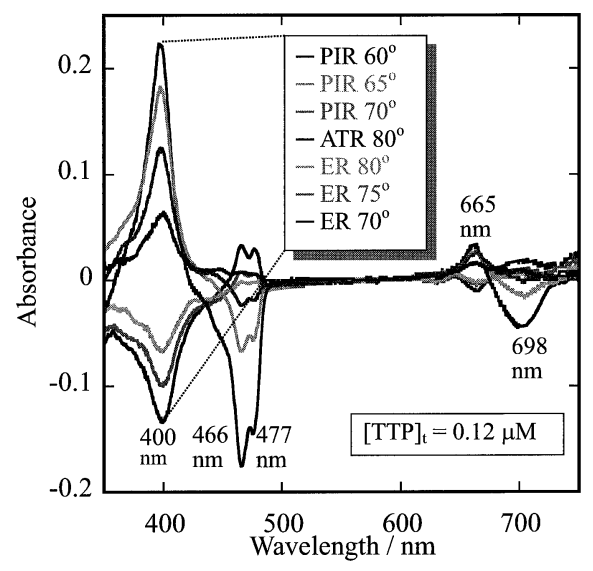

Fig. 6 Angle-dependent $p$-polarized reflection spectra.

the interfacial saturation. By considering an estimated value $\left(2.2 \mathrm{~nm}^{2}\right)$ of the molecular cross-section area of the $\mathrm{H}_{2} \mathrm{TTP}^{2+}$ species, the average value for the tilt angle of the pyrrole ring plane was roughly evaluated to be $48^{\circ}$, which is near to that of the $\mathrm{H}_{2} \mathrm{TPP}^{2+}$ species in the same liquid-liquid system. ${ }^{13}$

The $p$-polarized ER spectrum is useful for a semi-quantitative analysis of the molecular orientation at an air-solid, an air-liquid, ${ }^{18}$ or a liquid-liquid interface. ${ }^{12,13}$ The angledependent spectra were measured by using a variable-angle device under the experimental condition of an interfacial molecular density of $0.9 \times 10^{-10} \mathrm{~mol} \mathrm{~cm}{ }^{-2}$. Figure 6 shows the p-polarized spectra at several angles of incidence for ER $\left(80^{\circ}\right.$, $\left.75^{\circ}, 70^{\circ}\right)$, ATR $\left(80^{\circ}\right)$ and PIR $\left(70^{\circ}, 65^{\circ}, 60^{\circ}\right)$. It is natural that all bands in the ATR spectrum at $\theta_{\text {ATR }}=80^{\circ}\left(\right.$ ATR $\left.80^{\circ}\right)$ appear with positive absorbance. In the ER $80^{\circ}$ spectrum corresponding to the ATR $80^{\circ}$, the 400 and the $665 \mathrm{~nm}$ bands are observed with a comparable, but negative, absorbance. The ER absorbance in absolute quantity increased with decreasing $\theta_{\mathrm{ER}}$ toward the Brewster angles $\left(\theta_{\mathrm{B}, \mathrm{ER}}=45.9^{\circ}\right)$. It is noteworthy that both of the split bands at 477 and $466 \mathrm{~nm}$ appeared as positive bands in the ER spectrum. It can thus be presumed that both are attributed to a chromophore having an electron-transition moment parallel to the interface by employing the interface selection rule of ER spectrometry. ${ }^{18}$ When the long axis of the J-aggregate is parallel with an interface, the pyrrol ring plane in the aggregate should be oriented obliquely at the interface. ${ }^{12,13}$ This idea can 
be supported by the average value $\left(48^{\circ}\right)$ for the tilt angle of the pyrrole ring plane, evaluated from the saturated interfacial molecular density, though we have still come to analyze the molecular orientation of aggregates based on the angledependent ER spectrum. Internal reflection with an incident angle of $70^{\circ}\left(<\theta_{\mathrm{C}}\right)$ does not belong to total-internal reflection any longer, but belongs to PIR. As shown in Fig. 6, the spectra of PIR70 $0^{\circ}$ and that of ER70 show a vertically symmetric relation; that is, the positive-negative signs of all bands are reversed at the same band locations. The PIR absorbance in absolute quantity increased with decreasing $\theta_{\mathrm{PIR}}$ toward $\theta_{\mathrm{B}, \mathrm{PIR}}=$ $44.1^{\circ}$. The results obtained in the present experimental system strongly support the validity of our hypothesis concerning the interface selection rule in PIR spectrometry. ${ }^{13}$ That is, positive bands correspond to chromophores having a perpendicular electron-transition moment to the interface, while negative bands correspond to those having a parallel moment to the interface when the angle of incidence is within the region $\theta_{\mathrm{C}}>$ $\theta_{\mathrm{PIR}}>\theta_{\mathrm{B}, \mathrm{PIR}}$. For the region $0^{\circ}<\theta_{\mathrm{PIR}}<\theta_{\mathrm{B}, \mathrm{PIR}}$ the opposite is true. Thus, an appreciable analytical usefulness of partial reflection spectrometry was demonstrated by the measurement example of protonated lipophilic tetraphenylporphyrin derivatives in the dodecane-aqueous sulfuric acid system.

\section{Acknowledgements}

This work was supported by a Grant-in-Aid for Scientific Reserch (No. 13650865) from the Ministry of Education, Culture, Sports, Science and Technology, Japan.

\section{References and Notes}

1. Y. Onoe and H. Watarai, Anal. Sci., 1998, 14, 237.
2. P. R. Danesi, "Principles and Practices of Solvent Extraction, Part I, Fundamental Principles", ed. J. Rydberg, C. Musikas, and G. R. Choppin, 1992, Chap. 5, Marcel Dekker, New York, 157.

3. A. Nelson, Langmuir, 1996, 12, 2058.

4. H. Nagatani and H. Watarai, Anal. Chem., 1998, 70, 2860.

5. J. M. Perera, J. K. McCulloch, B. S. Murray, F. Grieser, and G. W. Stevens, Langmuir, 1992, 8, 366.

6. S. Tsukahara and H. Watarai, Chem. Lett., 1999, 89.

7. H. Watarai and F. Funaki, Langmuir, 1999, 12, 6717.

8. T. Takenaka and T. Nakanaga, J. Phys. Chem., 1976, 80, 475.

9. A. Ohashi and H. Watarai, Chem. Lett., 2001, 1238 and ibid, 2003, 218.

10. T. Uchida, A. Yamaguchi, T. Ina, and N. Teramae, J. Phys. Chem. B, 2000, 104, 12091 .

11. Y. Moriya, N. Ogawa, T. Kumabe, and H. Watarai, Chem. Lett., 1998, 221.

12. Y. Moriya, R. Amano, T. Sato, S. Nakata, and N. Ogawa, Chem. Lett., 2000, 556.

13. Y. Moriya, T. Hasegawa, K. Hayashi, M. Maruyama, S. Nakata, and N. Ogawa, Anal. Bioanal. Chem., 2003, 376, 374.

14. D. L. Akins, H. Zhu, and C. Guo, J. Phys. Chem., 1996, 100,5420 .

15. S. Okada and H. Segawa, J. Am. Chem. Soc., 2003, 125, 2792.

16. When inspected by a software for waveform separation, it turns out that the band group within the wavelength region of 300 to $500 \mathrm{~nm}$ and that of 600 to $750 \mathrm{~nm}$ may consist of at least five bands, respectively.

17. O. Ohno, Y. Kaizu, and H. Kobayashi, J. Chem. Phys., 1993, 99, 4128.

18. T. Hasegawa, Bunseki (in Japanese), 1998, 582. 